University of Wollongong

Research Online

Faculty of Engineering - Papers (Archive)

Faculty of Engineering and Information

Sciences

$1-1-2005$

\title{
Robust control framework for piezoelectric actuation systems in micro/ nano manipulation
}

\author{
Hwee Choo Liaw \\ Monash University \\ Denny Oetomo \\ Monash University \\ Bijan Shirinzadeh \\ Monash University \\ Gursel Alici \\ University of Wollongong, gursel@uow.edu.au
}

Follow this and additional works at: https://ro.uow.edu.au/engpapers

Part of the Engineering Commons

https://ro.uow.edu.au/engpapers/3264

\section{Recommended Citation}

Liaw, Hwee Choo; Oetomo, Denny; Shirinzadeh, Bijan; and Alici, Gursel: Robust control framework for piezoelectric actuation systems in micro/nano manipulation 2005, 1-6.

https://ro.uow.edu.au/engpapers/3264

Research Online is the open access institutional repository for the University of Wollongong. For further information contact the UOW Library: research-pubs@uow.edu.au 


\section{Robust Control Framework for Piezoelectric Actuation Systems in Micro/Nano Manipulation}

\author{
Hwee Choo Liaw, Denny Oetomo, Bijan Shirinzadeh \\ Robotics and Mechatronics Research Laboratory \\ Department of Mechanical Engineering \\ Monash University, Clayton, VIC 3800 \\ \{hwee.liaw, denny.oetomo, bijan.shirinzadeh\}@eng.monash.edu.au
}

\author{
Gursel Alici \\ School of Mechanical, Materials, \\ and Mechatronics Engineering \\ University of Wollongong, NSW 2522 \\ gursel@uow.edu.au
}

\begin{abstract}
Micro/nano manipulation has been identified as one of the key enabling technologies for many emerging challenges. Within this scope, piezoelectric actuators have played major roles in achieving the required nano-resolution motion. This paper proposes a robust control framework for piezoelectric actuation systems to follow specified motion trajectories. The basic concept associated with this methodology lies in the specification of a target performance and the robust control scheme formulation for piezoelectric actuation systems to ensure the convergence of the position tracking error to zero. This control methodology is attractive as its implementation requires only the knowledge of the estimated system parameters and their corresponding bounds, including bound of hysteresis and external disturbances. Feasibility study of the framework for piezoelectric actuation systems in micro/nano manipulation is described. Simulation results validated the suitability of the proposed control approach.
\end{abstract}

\section{INTRODUCTION}

Micro/nano manipulation has been identified as one of the key enabling technologies for many research challenges such as: biomedical engineering, micro/nano manufacturing and assembly, micro/nano robotics, micro surgery, and nanotechnology, to name a few. In achieving these ultra-precision tasks, piezoelectric systems have been identified as the key component in motion actuation. Having high stiffness, fast response, and physically unlimited resolution, there is no other competitor in the current available technology that could offer comparable properties. In recent years, the advancements in piezoelectric actuator (PEA) designs, sensing devices (capacitive sensors, strain gauges), and combined with multiaxis flexure stages [1] have enabled the progress towards the steadily growing area of micro/nano-technology.

One major drawback of the PEAs is the presence of highly nonlinear hysteretic behaviour between the input (applied) voltage and the output displacement. This prevents the PEA from providing the desired high-precision motion. A considerable amount of research has been conducted in this area to model and compensate for the hysteresis effect. Some examples include the modelling of physical hysteresis [2], dynamic model of hysteresis for a bi-morph beam [3], a comprehensive voltage-input electromechanical model [4], and a charge steering model that bypasses hysteretic problem coupled with comprehensive model of mechanical dynamics of the PEA [5].
Many approaches of control strategies have also been proposed in the past. Recent examples include an adaptive control using back-stepping approach [6], a combination of feedforward model with feedback control with an input shaper [7], PID-based control with iterative learning plus disturbance observer [8], and a sliding mode control augmented with inverse hysteresis model [9].

In this paper, a robust control framework is established for the PEA systems. This is motivated by the previous success in development of a high-precision linear motion system [10]. In this control framework, the variable structure control approach [11] is employed to deal with not only the parametric uncertainties but also the hysteresis of the PEA, including unmodelled disturbances. The proposed robust control methodology will steer a PEA system to closely follow a desired motion trajectory and drive the system to reach a specified target performance. Implementation of the system requires only the knowledge of the estimated parameters and their corresponding bounds including bound of hysteresis and external disturbances. Simulation conducted in Matlab-Simulink validated the feasibility of the proposed control approach. With the capability of handling uncertainties, the control scheme is very attractive in high performance PEA control applications, through which robust micro/nano-manipulation systems could be realised.

This paper is organized as follows. The model of a piezoelectric actuator is presented in Section II, a target performance is introduced in Section III, and modelling of uncertainties is described in Section IV. A robust control methodology is formulated in Section V and followed by simulation study in Section VI. The simulation results are shown and discussed in Section VII, and finally, conclusions are drawn in Section VIII.

\section{Model of Piezoelectric Actuator}

An electromechanical model of a PEA is given in [4], [5]. This mathematical model can be divided into three stages of transformation from electrical to mechanical energy, and vice versa. The schematic model as shown in Fig. 1 illustrates the transformation, which consists of voltage-charge, piezo, and force-displacement stages. Note that the model in Fig. 1 is formulated for a voltage-controlled amplifier. The dynamic equation from the electrical input to the output motion stage 


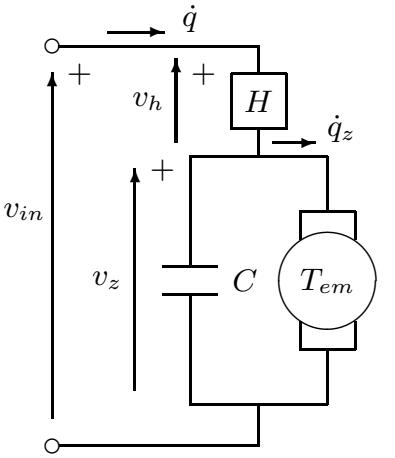

Voltage-Charge:

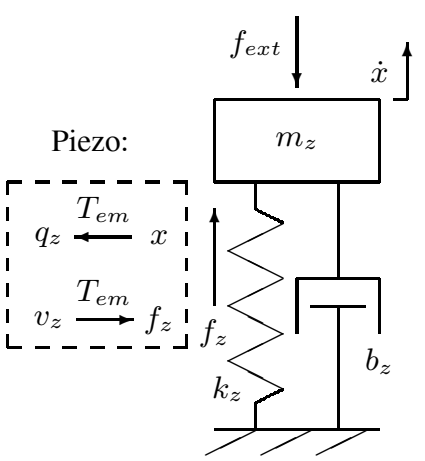

Force-Displacement:
Fig. 1. Schematic model of a piezoelectric actuator

can be described by the following set of equations:

$$
\begin{aligned}
v_{i n} & =v_{h}+v_{z}, \\
v_{h} & =H(q), \\
q & =C v_{z}+q_{z}, \\
q_{z} & =T_{e m} x, \\
f_{z} & =T_{e m} v_{z} \\
m_{z} \ddot{x}+b_{z} \dot{x}+k_{z} x & =f_{z}-f_{\text {ext }},
\end{aligned}
$$

where $v_{i n}$ represents the applied (input) voltage, $v_{h}$ is the voltage due to hysteresis, $v_{z}$ is the voltage related to mechanical side of the actuator, $q$ is the total charge in the ceramic, $H$ is the hysteresis effect, $C$ is the linear capacitance connected in parallel with the electromechanical transformer having a ratio of $T_{e m}, q_{z}$ is the piezo charge related to the actuator output displacement $x, f_{z}$ is the transduced force from the electrical domain, $m_{z}, b_{z}$, and $k_{z}$ are the mass, damping, and stiffness, respectively, of the mechanical stage, and $f_{\text {ext }}$ is the force imposed by the external mechanical load. In PEA, hysteresis causes a highly nonlinear input/output relationship between the applied voltage and displacement. Goldfarb and Celanovic [4] described the hysteresis effect as a nonlinear charge-dependent phenomenon and noted that it appeared only in the electrical domain.

For control purposes, (1) and (5) are substituted into (6) to yield

$$
m_{z} \ddot{x}+b_{z} \dot{x}+k_{z} x=T_{e m}\left(v_{i n}-v_{h}\right)-f_{\text {ext }},
$$

and the PEA model is obtained by re-arranging the above,

$$
m \ddot{x}+b \dot{x}+k x+v_{h}+f_{e}=v_{i n},
$$

where $m=m_{z} / T_{e m}, b=b_{z} / T_{e m}, k=k_{z} / T_{e m}$, and $f_{e}=$ $f_{\text {ext }} / T_{\text {em }}$.

\section{TARget PERFormanCE}

In control of a system, it is desired that the system is commanded to follow a specified motion trajectory with a desired goal, which is defined as the target performance.

It is assumed that there exists an ideal model of (8), given as:

$$
m_{d} \ddot{x}+b_{d} \dot{x}+k_{d} x+v_{h}+f_{e}=v_{i n},
$$

where $m_{d}, b_{d}$, and $k_{d}$ are the desired constant values of mass, damping, and stiffness of the system, respectively, and the $v_{h}$ and $f_{e}$ are known. The system (9) is commanded to follow a desired motion trajectory $x_{d}(t)$ with a desired command,

$$
v_{d}=m_{d} \ddot{x}_{d}+b_{d} \dot{x}_{d}+k_{d} x_{d}+v_{h}+f_{e} .
$$

If the desired command in (10) is exactly the same as applied voltage in (9), i.e. $v_{d}=v_{i n}$, then the target performance can be formulated by combining these equations and is given by the error function:

$$
m_{d} \ddot{e}_{p}+b_{d} \dot{e}_{p}+k_{d} e_{p}=0,
$$

where $e_{p}(t)=x(t)-x_{d}(t)$. With suitable choice of $m_{d}, b_{d}$, and $k_{d}$, the error function (11) is stable (i.e. $e_{p} \rightarrow 0$ ) and describes the closed loop dynamics of the system.

\section{Modelling of UnCertainties}

In practice, the exact parameters of the system (8) are difficult, if not impossible, to determine. The modelling of parametric uncertainties is therefore introduced. It is stated that the exact values of $m, b$, and $k$ in (8) may not be known, however the estimated values are available. The modelling of uncertainties can also be extended to include the nonlinear hysteresis in (8) and it is assumed that both the parametric errors and hysteresis are bounded:

$$
\begin{aligned}
\|\Delta m\| & =\|m-\hat{m}\| \leq \delta m \\
\|\Delta b\| & =\|b-\hat{b}\| \leq \delta b \\
\|\Delta k\| & =\|k-\hat{k}\| \leq \delta k \\
\left\|v_{h}\right\| & \leq \delta v_{h},
\end{aligned}
$$

where $\Delta \bullet$ represents the modelling error of $\bullet$ and $\hat{\bullet}$ represents the estimated values of $\bullet$. The symbol $\|\bullet\|$ denotes an absolute value of $\bullet$ and the positive values $\delta m, \delta b, \delta k$, and $\delta v_{h}$ denote the bounds of the variables. These bounds are known and can be obtained experimentally. Generally, the bound $\delta v_{h}$ could be further extended to incorporate other un-modelled disturbances in the system provided that the overall bound of the uncertainties is known.

\section{Robust Control Methodology}

The motion control problem in the PEA system can be formulated as a target performance reaching problem in designing a control law so that the system described by (8) achieves the target performance (11) and follows the required trajectory $x_{d}(t)$ under parametric uncertainties and hysteresis (12). In the following, a robust control methodology is formulated to solve the problem.

To achieve robust control, a switching function $\sigma$ is first specified,

$$
\sigma=\dot{e}_{p}+\xi
$$

where $\xi$ is the state of a dynamic compensator used to shape the tracking errors. The dynamic compensator can be designed as

$$
\dot{\xi}=\alpha \xi+k_{p} e_{p}+k_{v} \dot{e}_{p}
$$


where $\alpha$ is a constant scalar, $\alpha \leq 0, k_{p}$ and $k_{v}$ are constant values which are related to the specified target performance (11). Differentiating (13) with respect to time,

$$
\dot{\sigma}=\ddot{e}_{p}+\dot{\xi} .
$$

To examine the closed loop dynamics of the system under the sliding mode control, equation (14) is substituted into (15) and using (13) to eliminate $\xi$,

$$
\ddot{e}_{p}+\left(k_{v}-\alpha\right) \dot{e}_{p}+k_{p} e_{p}=\dot{\sigma}-\alpha \sigma .
$$

By choosing

$$
\begin{aligned}
& k_{p}=m_{d}^{-1} k_{d}, \\
& k_{v}=m_{d}^{-1} b_{d}+\alpha,
\end{aligned}
$$

(16) becomes

$$
m_{d} \ddot{e}_{p}+b_{d} \dot{e}_{p}+k_{d} e_{p}=m_{d}(\dot{\sigma}-\alpha \sigma) .
$$

During sliding motion where $\dot{\sigma}=0$ and $\sigma=0$, (18) achieves the target performance (11). A control law can therefore be formulated to drive the system to reach the sliding mode.

Theorem: For the system described by (8) under parametric uncertainties and hysteresis (12), the system achieves the target performance (11) with the following robust control law

$$
v_{i n}=\hat{m} \ddot{x}_{e q}+\hat{b} \dot{x}+\hat{k} x+f_{e}-k_{s} \sigma-d \frac{\sigma}{\|\sigma\|},
$$

where

$$
\ddot{x}_{e q}=\ddot{x}_{d}-\dot{\xi},
$$

and $d$ is governed by

$$
d \geq \delta m\left\|\ddot{x}_{e q}\right\|+\delta b\|\dot{x}\|+\delta k\|x\|+\delta v_{h}+\epsilon,
$$

where $k_{s}$ and $\epsilon$ are any positive scalars.

Proof: For the system described by (8) with the control law (19), a Lyapunov function $u(\sigma)$ is proposed,

$$
u(\sigma)=\frac{1}{2} m \sigma^{2},
$$

which is continuous and non-negative. Differentiating $u(\sigma)$ with respect to time yields

$$
\dot{u}(\sigma)=m \sigma \dot{\sigma} .
$$

From (15) and (20),

$$
\dot{\sigma}=\ddot{x}-\ddot{x}_{e q},
$$

and (23) is rewritten as

$$
\begin{aligned}
\dot{u}(\sigma) & =\sigma\left(m \ddot{x}-m \ddot{x}_{e q}\right), \\
& =\sigma\left(v_{i n}-b \dot{x}-k x-v_{h}-f_{e}-m \ddot{x}_{e q}\right)
\end{aligned}
$$

where $v_{i n}$ is obtained from (8). Substituting the control law (19) to replace $v_{i n}$ and using the bounds (12),

$$
\begin{aligned}
\dot{u}(\sigma)= & -k_{s} \sigma^{2}-d\|\sigma\|+\sigma\left[-\Delta m \ddot{x}_{e q}-\Delta b \dot{x}\right. \\
& \left.-\Delta k x-v_{h}\right], \\
\leq & -k_{s} \sigma^{2}-d\|\sigma\|+\|\sigma\|\left[\left\|\Delta m \ddot{x}_{e q}\right\|+\|\Delta b \dot{x}\|\right. \\
& \left.+\|\Delta k x\|+\left\|v_{h}\right\|\right], \\
\leq & -k_{s} \sigma^{2}-d\|\sigma\|+\|\sigma\|\left[\delta m\left\|\ddot{x}_{e q}\right\|+\delta b\|\dot{x}\|\right. \\
& \left.+\delta k\|x\|+\delta v_{h}\right] .
\end{aligned}
$$

From (21),

$$
\dot{u}(\sigma) \leq-k_{s} \sigma^{2}-\epsilon\|\sigma\| .
$$

This shows that $u(\sigma) \rightarrow 0$ (which in turn implies that $\sigma \rightarrow 0$ ) as $t \rightarrow \infty$. Both the stability of the system and convergence of the tracking are guaranteed by the robust control law (19) driving the system (8) to reach the target performance (11).

In the implementation of the control law (19), the discontinuous function $\frac{\sigma}{\|\sigma\|}$ will give rise to control chattering due to imperfect switching in the computer control. This is undesirable as un-modelled high frequency dynamics might be excited. To eliminate this effect, the concept of boundary layer technique [12] is applied to smooth the control signal. In a small neighborhood of the sliding surface $(\sigma=0)$, the discontinuous function is replaced by a saturation function which is defined as

$$
\operatorname{sat}\left(\frac{\sigma}{\Delta}\right)=\left\{\begin{array}{lll}
-1 & : & \sigma<-\Delta \\
\sigma / \Delta & : & -\Delta \leq \sigma \leq \Delta \\
+1 & : & \sigma>\Delta
\end{array}\right.
$$

where $\Delta$ is the boundary layer thickness, and the control law (19) becomes

$$
v_{i n}=\hat{m} \ddot{x}_{e q}+\hat{b} \dot{x}+\hat{k} x+f_{e}-k_{s} \sigma-d \operatorname{sat}\left(\frac{\sigma}{\Delta}\right) .
$$

With the introduction of the saturation function (28) in the control law (29), the accuracy of $\sigma$ can only be guaranteed to stay within the boundary layer. From the closed loop dynamics (18) of the control law, the steady-state value, $\sigma_{s s}$, of the switching function within the boundary layer is given as

$$
\sigma_{s s}=-\frac{k_{d} e_{p s s}}{m_{d} \alpha}
$$

where $e_{p s s}$ is the steady-state position error. As (30) describes the relationship between the steady-state position error and switching function, it can therefore be used to decide on the boundary layer thickness $\Delta$ in the control implementation.

The selection of target performance for the control system is straightforward. By comparing (11) to a standard second-order characteristic equation

$$
s^{2}+2 \zeta w_{n} s+w_{n}^{2}=0,
$$

where $s, \zeta$, and $w_{n}$ are the Laplace operator, damping ratio, and undamped natural frequency, respectively, the desired parameters are obtained as

$$
m_{d}=1, \quad b_{d}=2 \zeta w_{n}, \quad k_{d}=w_{n}^{2} .
$$

As the desired response is selected through $\zeta$ and $w_{n}$, the system control gains $k_{p}$ and $k_{v}$ in (17) can therefore be calculated from (32).

\section{Simulation Study}

In the process of developing a PEA system for micro/nano manipulation, computer simulation is performed to study the feasibility of the proposed control strategy. The simulation model is constructed using the PEA model (8) and the control 

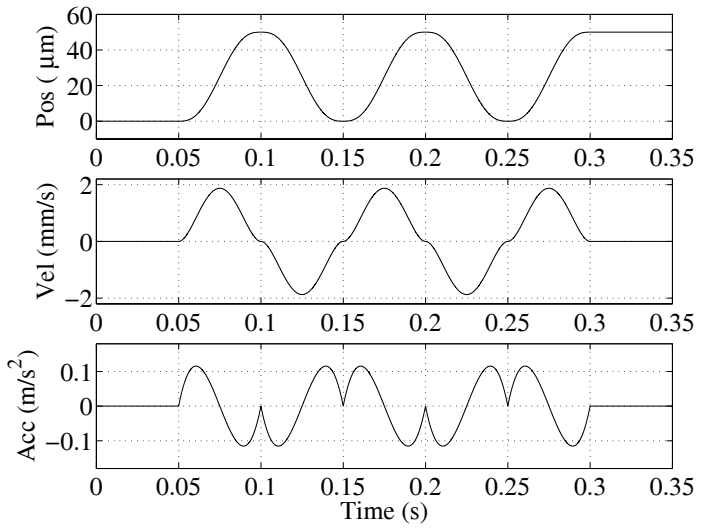

Fig. 3. Desired motion trajectory

law with saturation function (29). This is conducted in Matlab [13] Simulink [14] as shown in Fig. 2.

The objective of the simulation is to study the effectiveness of the proposed control algorithm such that the closed loop method will follow a motion trajectory with a desirable tracking ability. In the simulation, the proposed desired motion trajectory is shown in Fig. 3 for position, velocity, and acceleration, respectively. The desired motion trajectory is made up of different segments of quintic polynomial [15] for analysing the tracking and steady-state performances of the system. With the motion trajectory, the tracking ability of the control system can be closely examined when it is subjected to parametric uncertainties and hysteresis effects.

For a more realistic simulation, a model of hysteresis is added to the ideal PEA model as shown in Fig. 2. As described in (2), the PEA hysteresis is dependent on the charge $q$, which is constituted by the useful piezo charge $q_{z}$ and charge in the capacitor $C$ as shown in Fig. 1. As $q_{z}$ is related to the actual motion $x$ in (4) and by assuming that $x$ follows closely the desired position $x_{d}$, approximated polynomial functions are utilised to construct the hysteresis model, which are dependent on $x_{d}$. The hysteresis $v_{h}$ of the PEA model (8) is approximated by

$v_{h f}(t)=\left(x_{d}-\left(5 \times 10^{-6}+0.4 x_{d}+8000 x_{d}^{2}\right)\right) \times 10^{6} V$,
$v_{h b}(t)=\left(x_{d}-\left(5 \times 10^{-6}+1.5 x_{d}-14000 x_{d}^{2}\right)\right) \times 10^{6} V$,

where $v_{h f}$ and $v_{h b}$ are the voltage-drops representing the hysteresis effect when the PEA is moving forward or backward, respectively. The resulting effect of the hysteresis model is shown in Fig. 4.

It is assumed that no external force is applied to the system and the term $f_{e}$ is ignored in both (8) and (29). However, a constant bias is added in the simulation to offset the command voltage to operate only in the positive region. Alternatively, the bias can be treated as to represent a preloaded force to the PEA in reality.

Table I summarises the values chosen for the simulation. The PEA model (8) was simulated to possess dynamic coefficients as shown in the first column (labelled "Exact Values")

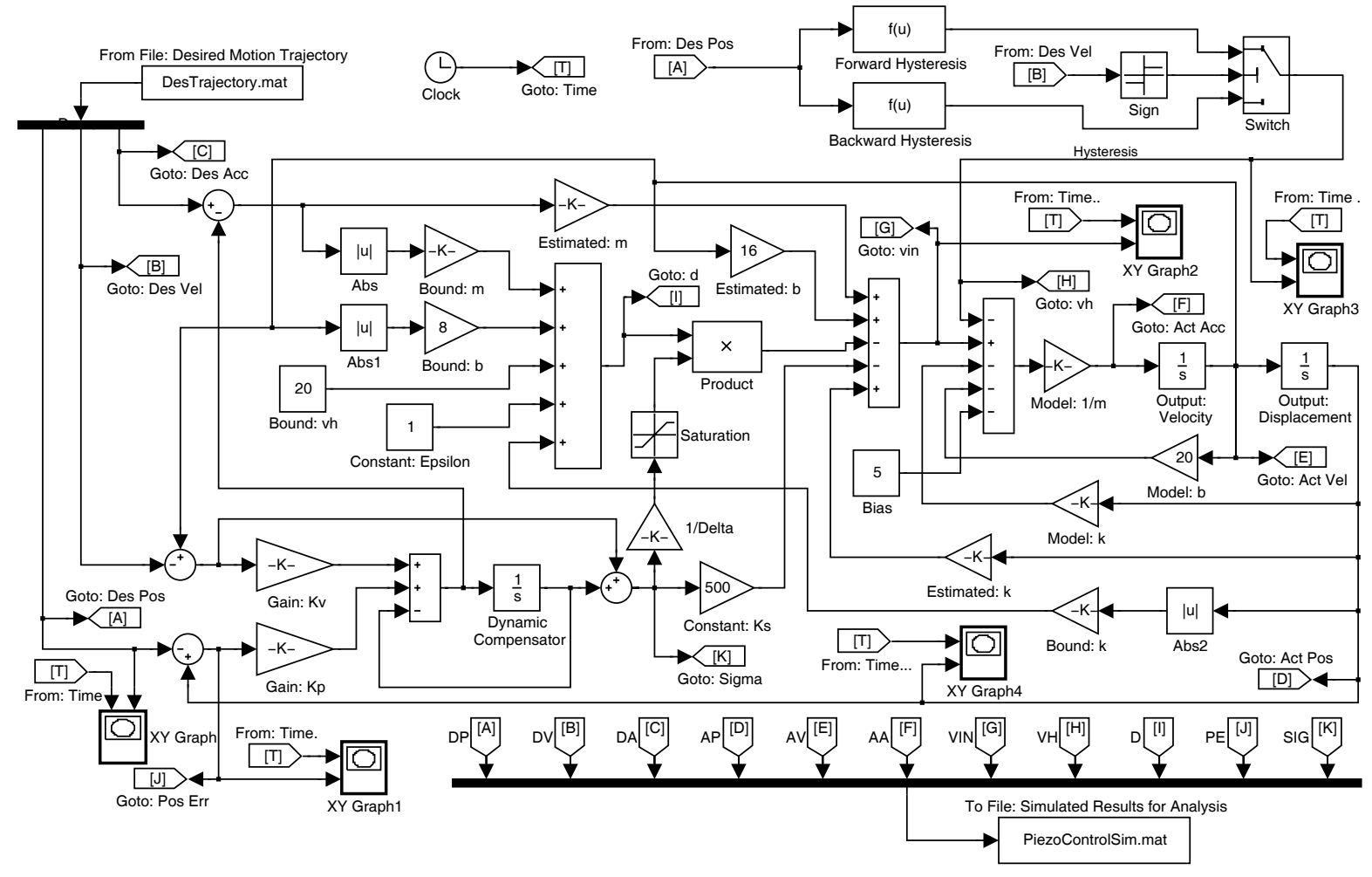

Fig. 2. Simulation of piezoelectric actuation system in Matlab-Simulink 


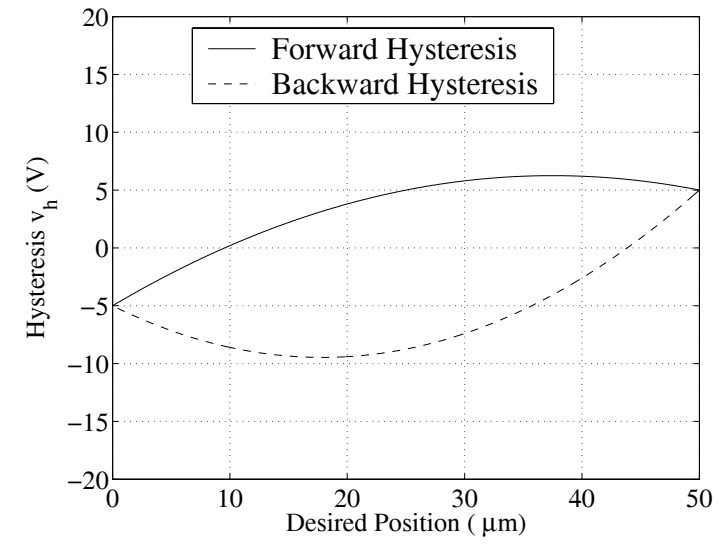

Fig. 4. Hysteresis model

TABLE I

PARAMETERS FOR SIMULATION OF PEA CONTROL SYSTEM

\begin{tabular}{|l|l|l|l|}
\hline & Exact Values: & Est. Values: & Bounds: \\
\hline Mass $\left(V s^{2} / m\right):$ & $m=0.0005$ & $\hat{m}=0.0004$ & $\delta m=0.0002$ \\
\hline Damping $(V s / m):$ & $b=20$ & $\hat{b}=16$ & $\delta b=8$ \\
\hline Stiffness $(V / m):$ & $k=5 \times 10^{5}$ & $\hat{k}=4 \times 10^{5}$ & $\delta k=2 \times 10^{5}$ \\
\hline Hysteresis $(V):$ & & & $\delta v_{h}=20$ \\
\hline
\end{tabular}

of the table. Assuming that only the estimated parameters and bounds are available for the control law (29). The estimated dynamic coefficients are listed in the second column (labelled "Est. Values") and the bounds of uncertainties are quantified and listed in the third column. Note that the bound of the hysteretic disturbance is also included in Table I according to Fig. 4.

For an undamped natural frequency of $w_{n}=1256.64 \mathrm{rad} / \mathrm{s}$ $(200 \mathrm{~Hz})$ and a critically damped response, $\zeta=1.0$, the desired parameters in (32) are calculated as

$m_{d}=1 \mathrm{Vs}^{2} / \mathrm{m}, b_{d}=2513 \mathrm{Vs} / \mathrm{m}$, and $k_{d}=1579,137 \mathrm{~V} / \mathrm{m}$.

The constant scalar $\alpha$ in (14) is set as $\alpha=-1 s^{-1}$ and the control gains $k_{p}$ and $k_{v}$ in (17) are calculated from (32) as

$$
k_{p}=1579,137 \mathrm{~s}^{-2} \text { and } k_{v}=2512 \mathrm{~s}^{-1} .
$$

The steady-state position error in (30) is specified as $e_{p s s} \leq$ $0.1 \mu \mathrm{m}, \sigma_{s s} \leq 0.16 \mathrm{~m} / \mathrm{s}$, the boundary layer thickness $\Delta$ in (28) is chosen as the maximum value of $\sigma_{s s}$, i.e., $\Delta=$ $0.16 \mathrm{~m} / \mathrm{s}$.

The positive scalar $\epsilon$ in (21) is specified as $\epsilon=1 \mathrm{~V}$ and $k_{s}$ of the control law (29) is set to $k_{s}=500 \mathrm{Vs} / \mathrm{m}$.

\section{RESULTS AND DiscUSSION}

In Simulink, a Runge-Kutta 45 based ordinary differential equation solver with variable step size was employed for numerical integration of the acceleration to compute the velocity and displacement of the PEA model. Simulated results are shown in Fig. 5 to Fig. 8.
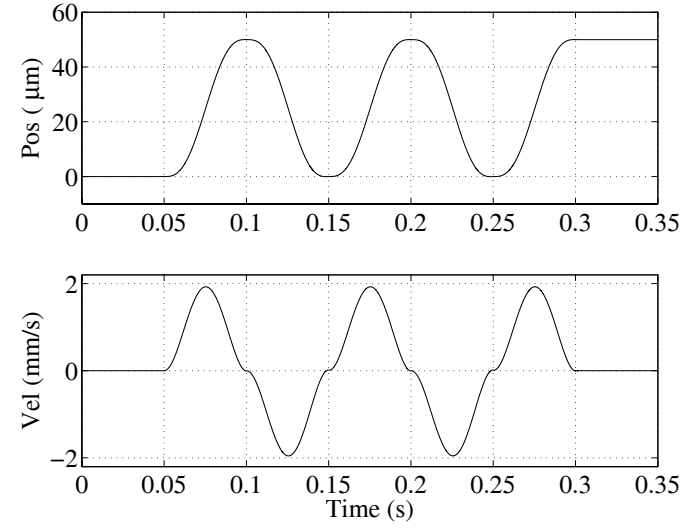

Fig. 5. Resulted PEA motion trajectory
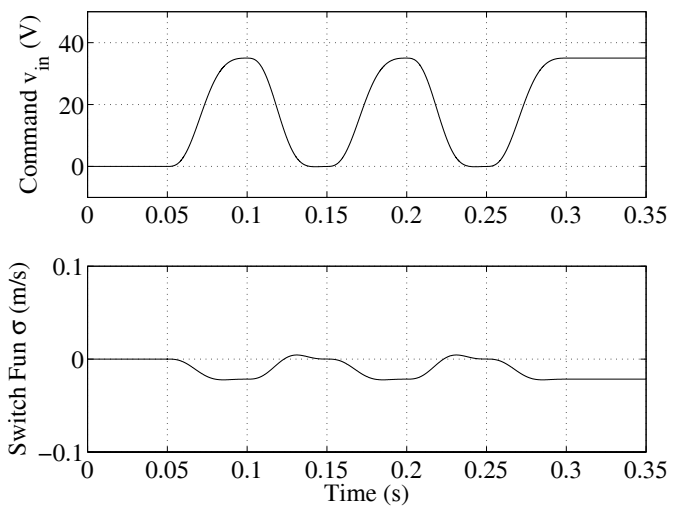

Fig. 6. Command voltage and switching function

With the desired trajectory as shown in Fig. 3, the resulting motion is shown in Fig. 5. Despite the uncertainties, the control law was shown to be robust and stable. From the switching function shown in Fig. 6, it was observed that the system operated within the boundary layer with $\sigma$ kept to a minimum.

The hysteresis effect and position tracking error are shown in Fig. 7. The position tracking result showed that the control law had successfully accommodated the hysteresis, without any feed-forward model, treating the hysteresis purely as an external disturbance. While the disturbance caused by the hysteresis was significant $\left(v_{h}\right.$ fluctuated from approximately $-10 \mathrm{~V}$ to $6 \mathrm{~V}$ - see Fig. 7 top), forming about one-third of the reference (command) voltage ( $v_{\text {in }}$ ranged from 0 to $35 \mathrm{~V}$ - see Fig. 6), the effect of the resulting position tracking error in (Fig. 7 bottom) was small compared to the disturbances. In the simulation, the position tracking error was confined within $1 \mu \mathrm{m}$ during motion in the displacement range of $50 \mu \mathrm{m}$ (see Fig. 8) and the steady-state error was almost zero. Fig. 8 shows the resulting actuator position when plotted against the desired position with a minimum hysteresis effect. This showed the effectiveness of the proposed robust control framework.

On the whole, the robust control methodology was shown to be stable, robust and capable of following the desired motion trajectory under modelling of parametric uncertainties and hysteresis. The control implementation required only the 

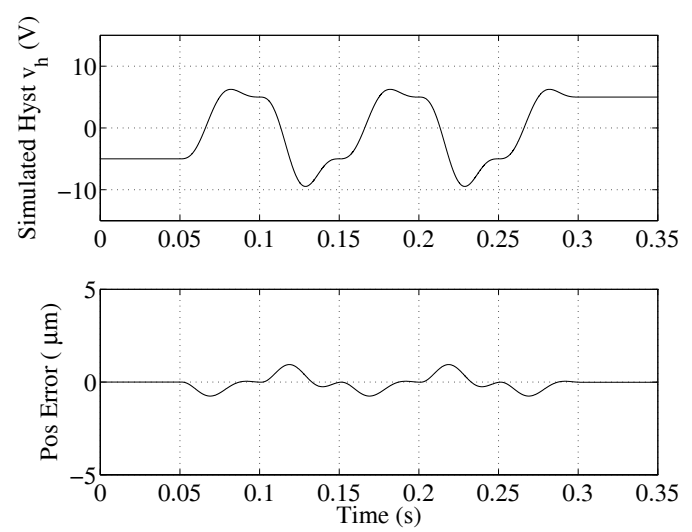

Fig. 7. Hysteresis effect and position tracking error

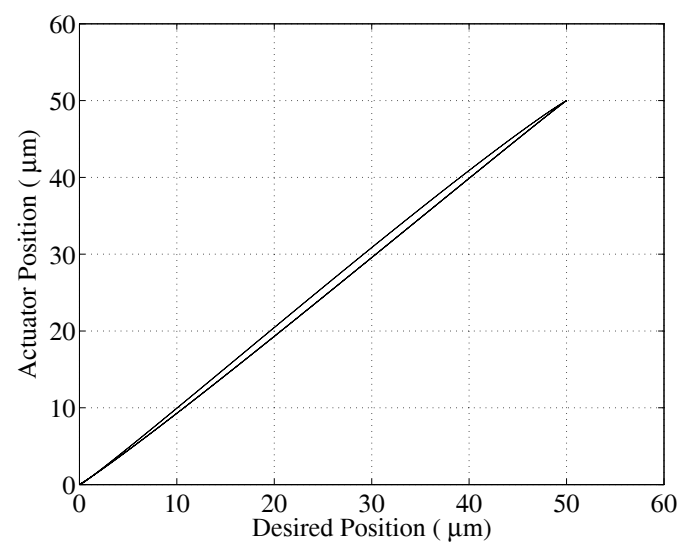

Fig. 8. Actuator position against desired position

estimated parameters and the bounds of the system.

For this methodology to be implemented on a physical system, it is necessary to examine the issue of parameter identification and controller realisation. Challenges lie in the fact that micro/nano manipulation systems require different strategies from conventional motion control. An experimental set-up is being prepared to implement the algorithm through a hard-real time system involving the PEA system driving a parallel micro/nano manipulator based on flexure-jointed monolithic structure. Position feedback will be provided by non-contact capacitive sensors capable of sub-nanometer resolution. The result will form an important comparison to the theoretical prediction and enhance our understanding towards robust micro/nano manipulation.

\section{CONCLUSIONS}

A robust control framework is proposed for piezoelectric actuation systems to follow specified motion trajectories. The basic concept in this approach lies in the specification of a target performance and the formulation of a robust control law for a control system to drive its position tracking error to converge to zero.

This control scheme is unique as a dynamic compensator is introduced to form the switching function such that the resulted sliding mode can be matched with the target performance. Implementation of the control law requires only the knowledge of the estimated parameters and their corresponding bounds as well as bound of hysteresis in the actuator including external disturbance.

Stability of the system is proven theoretically and the control scheme is shown to be robust through simulation. The immediate future effort is to implement this control scheme onto a PEA system. An extensive experimental set-up is being developed at this stage and it is expected that the experimental results on the real system be available in the near future.

Being capable of handling uncertainties, the robust control scheme is very attractive in the field of micro/nanomanipulation in which high performance PEA control applications could be implemented specifically in the biomedical area.

\section{ACKNOWLEDGMENT}

This work is supported by ARC LIEF grant, ARC Discovery grant, and Monash Research Fund.

\section{REFERENCES}

[1] K. Spanner and S. Vorndran, "Advances in piezo-nanopositioning technology," in Proc. IEEE/ASME International Conference on Advanced Intelligent Mechatronics, vol. 2, Kobe, Japan, 20-24 July 2003, pp. 1338-1343.

[2] Y. I. Somov, "Modelling physical hysteresis and control of a fine piezodrive," in Proc. International Conference Physics and Control, vol. 4, St. Petersburg, Russia, 20-22 August 2003, pp. 1189-1194.

[3] T. S. Low and W. Guo, "Modeling of a three-layer piezoelectric bimorph beam with hysteresis," Journal of Microelectromechanical Systems, vol. 4, no. 4, pp. 230-237, December 1995.

[4] M. Goldfarb and N. Celanovic, "Modeling piezoelectric stack actuators for control of micromanipulation," IEEE Control Systems Magazine, vol. 17, no. 3, pp. 69-79, June 1997.

[5] H. J. M. T. A. Adriaens, W. L. D. Koning, and R. Banning, "Modeling piezoelectric actuators," IEEE/ASME Transactions on Mechatronics, vol. 5, no. 4, pp. 331-341, December 2000.

[6] H. J. Shieh, F. J. Lin, P. K. Huang, and L. T. Teng, "Adaptive tracking control solely using displacement feedback for a piezo-positioning mechanism," IEE Proc. Control Theory and Applications, vol. 151, no. 5, pp. 653-660, September 2004.

[7] T. Chang and X. Sun, "Analysis and control of monolithic piezoelectric nano-actuator," IEEE Transactions on Control Systems Technology, vol. 9, no. 1, pp. 69-75, January 2001.

[8] Y. C. Huang and C. H. Cheng, "Robust tracking control of a novel piezodriven monolithic flexure-hinge stage," in Proc. IEEE International Conference on Control Applications, vol. 2, Taipei, Taiwan, 2-4 September 2004, pp. 977-982.

[9] S. Yu, B. Shirinzadeh, G. Alici, and J. Smith, "Sliding mode control of a piezoelectric actuator with neural network compensating rate-dependent hysteresis," in Proc. IEEE International Conference on Robotics and Automation, Barcelona, Spain, 18-22 April 2005, pp. 3652-3656.

[10] H. C. Liaw, S. Y. Lim, W. Lin, and K. K. Tan, "Robust model-reaching control of high precision linear motion systems," in Proc. 29th Annual Conference of IEEE Industrial Electronics Society, Roanoke, Virginia, 2-6 November 2003, pp. 2794-2799.

[11] J. Y. Hung, W. B. Gao, and J. C. Hung, "Variable structure control: a survey," IEEE Transactions on Industrial Electronics, vol. 40, no. 1, pp. 2-22, 1993.

[12] J. J. E. Slotine and W. Li, Applied Nonlinear Control. Englewood Cliffs, NJ: Prentice-Hall, 1991.

[13] (2005) The mathworks. [Online]. Available: http://www.mathworks.com/

[14] Using Simulink: Version 5. Natick, MA: The MathWorks, 2003.

[15] J. J. Craig, Introduction to Robotics: Mechanics and Control. Reading, MA: Addison-Wesley, 1989. 